\title{
Urinary excretion of the hydroxypyridinium cross links of collagen in patients with rheumatoid arthritis
}

\author{
D BLACK, ${ }^{1}$ M MARABANI ${ }^{2}$ R D STURROCK, ${ }^{2}$ AND S P ROBINS
}

From the ${ }^{1}$ Rowett Research Institute, Bucksburn, Aberdeen; and the ${ }^{2}$ Centre for Rheumatic Diseases, The Royal Infirmary, Glasgow

SUMMARY Values for urinary hydroxypyridinium cross links of collagen measured by high performance liquid chromatography in a group of 19 seropositive patients with rheumatoid arthritis (RA) were compared with 19 controls matched for age and sex. The amounts of pyridinoline, expressed relative to creatinine, were significantly higher in the RA group, but there was no significant increase in a bone specific analogue, deoxypyridinoline. There were significant positive correlations between pyridinoline excretion and both $\mathrm{C}$ reactive protein and erythrocyte sedimentation rate, whereas there was an inverse relation with grip strength. The data indicated that pyridinoline concentrations in urine are strongly associated with disease activity in patients with RA.

It has been proposed that the cross links of mature collagen, pyridinoline, and its analogue, deoxypyridinoline, may provide satisfactory biochemical indices that reflect the breakdown of mature collagen, and thus be potentially useful clinical markers for the progression and activity of inflammatory disturbances of the extracellular matrix. ${ }^{12}$ Pyridinoline is formed non-enzymatically, either by the reaction of two bifunctional keto-amine cross links ${ }^{3}$ or by the condensation of hydroxylysine aldehyde with an existing bifunctional keto-amine cross link. ${ }^{4}$ This component is found predominantly in type $I$ and type II collagens of bone and cartilage respectively, but not in type I collagen of skin. ${ }^{3}$ Deoxypyridinoline, however, is formed from the reaction of lysine rather than hydroxylysine as the amino group donor and appears to be located only in the type I collagen of bone and dentine. ${ }^{5}$ Both cross links are excreted in the urine ${ }^{2}$ so that measurement provides an estimate of the degradation of mature, insoluble collagen from specific tissues. Thus unlike other commonly used biochemical markers for collagen degradation, such as urinary hydroxyproline and hydroxylysine glycosides, these measurements are free from the interference resulting from the breakdown of collagen precursors or from other proteins containing collagen-like sequences, such as

Accepted for publication 13 January 1989

Correpondence to Dr S P Robins, Rowett Research Institute, Greenburn Road, Bucksburn, Aberdeen AB2 9SB. complement $\mathrm{C} 1 \mathrm{q} .{ }^{6}$ The latter is of course significantly raised in inflammatory disorders.

An enzyme linked immunoassay (ELISA) for pyridinoline was developed ${ }^{1}$ and used to show increased urinary excretion of this component in patients with osteoarthritis and rheumatoid arthritis (RA). ${ }^{7}$ The deoxy derivative was, however, unreactive with the antibody, and a high performance liquid chromatography (HPLC) technique to measure both cross links simultaneously has now been developed. ${ }^{2}$ To assess the value of the hydroxypyridinium cross links as clinical markers of disease activity the present study was carried out in which the urinary levels of both cross links in patients with classical RA were compared with recognised clinical indices routinely used to measure activity of the disease.

\section{Patients and methods}

Nineteen patients with definite or classical RA by American Rheumatism Association criteria, admitted consecutively to the rheumatology ward at Glasgow Royal Infirmary, were studied. Patients over 65 years of age were excluded as were those with renal impairment, diabetes, or those receiving systemic steroids. All of the RA group were taking non-steroidal anti-inflammatory drugs and some were also undergoing second line or cytotoxic treatment: no attempt was made to standardise treatment during the study. Healthy volunteers, 
matched for age and sex, and with no history of articular disease served as controls.

The following clinical and laboratory indices were estimated in the patients with RA: duration of morning stiffness, grip strength using an electric dynamometer (MIE Medical Research Ltd, Aylesbury, Bucks, UK), articular index, erythrocyte sedimentation rate (ESR) and $C$ reactive protein (CRP) with a semi-automated IRMA TDX kit from Abbot Laboratories (Sheerness, Kent, UK); the coefficient of variation for the assay was $<10 \%$. In addition, $x$ ray examinations of the hands were made and graded according to a modification of Sharp's method. ${ }^{8}$

Samples of urine were collected for 24 hours, the volume noted, and an aliquot removed and stored at $-20^{\circ} \mathrm{C}$ until analysis. Pyridinoline and deoxypyridinoline were measured in an aliquot of hydrolysed urine by HPLC as described previously. ${ }^{2}$ Briefly, $250 \mu \mathrm{l}$ of urine were hydrolysed in an equal volume of concentrated $\mathrm{HCl}$ for 18 hours at $107^{\circ} \mathrm{C}$. The hydrolysates were fractionated by CFI cellulose partition chromatography to remove interfering fluorescent components, and an aliquot of the appropriate fraction was analysed for the hydroxypyridinium cross links using $C_{18}$ reversed phase HPLC with fluorimetric detection. The results were expressed as nmol cross link/mmol creatinine, the latter being measured in unhydrolysed portions of the urine. ${ }^{9}$

\section{Results}

Table 1 shows the clinical and laboratory features of the patients with RA, and it can be seen that the group studied was heterogeneous with respect to age, grip strength, ESR, and CRP. Nevertheless, the median values of early morning stiffness, ESR, and CRP indicate significant disease activity in this group of individuals.

The urinary concentrations of pyridinoline, expressed as a pyridinoline/creatinine ratio, were significantly higher $(p<0.001)$ than those in the 19

Table 1 Clinical and laboratory data for the patients with rheumatoid arthritis in the study

\begin{tabular}{lcl}
\hline & Median & (Range) \\
\hline Age & 53 & $(24-65)$ \\
Early morning stiffness $(\mathrm{min})$ & 120 & $(0-720)$ \\
Articular index & 18 & $(0-37)$ \\
Grip strength $(\mathrm{mmHg})$ & 37 & $(10-107)$ \\
ESR $^{*}(\mathrm{~mm} / \mathrm{h})$ & 68 & $(4-128)$ \\
CRP $^{*}(\mathrm{~g} / \mathrm{l})$ & $0 \cdot 49$ & $(0 \cdot 13-1 \cdot 56)$ \\
\hline
\end{tabular}

${ }^{*} \mathrm{ESR}=$ erythrocyte sedimentation rate; $\mathrm{CRP}=\mathrm{C}$ reactive protein.
Table 2 Hydroxypyridinium cross links in the urine of $\frac{\square}{5}$ patients with rheumatoid arthritis $(R A)$ compared with age and sex matched controls. Mean values $(S D)$ are expressed $\overrightarrow{\bar{\sigma}}$ as nmol cross link/mmol creatinine

\begin{tabular}{lll}
\hline & $\begin{array}{l}\text { Pyridinoline } \\
\text { (nmol/mmol } \\
\text { creatinine) }\end{array}$ & $\begin{array}{l}\text { Deoxy- } \\
\text { pyridinoline } \\
\text { (nmol/mmol } \\
\text { creatinine) }\end{array}$ \\
\hline $\begin{array}{l}\text { Age and sex matched controls } \\
\text { Patients with RA }\end{array}$ & $\begin{array}{l}27.2(8.2) \\
\text { Controls } v \text { patients with RA }\end{array}$ & $\begin{array}{l}11.0(78.9) \\
\mathrm{p}<0.001\end{array}$ \\
\hline
\end{tabular}

controls (Table 2). The concentrations of urinary ${ }_{\infty}^{\infty}$ deoxypyridinoline, however, were not significantly different from those of the controls and were within $\vec{A}$ the range established previously for normal indivi-음 duals $(8.4$ (SD 4.5$) \mathrm{nmol} / \mathrm{mmol}$ creatinine).

A direct comparison was made between urinary? pyridinoline and CRP, ESR, and grip strengtheo (Fig. 1). Significant positive correlations were noted $\stackrel{\text { s }}{9}$ between urinary pyridinoline and CRP $(r=0.786 ; \overrightarrow{0}$ $\mathrm{p}<0.001)$ and $\operatorname{ESR}(\mathrm{r}=0.691 ; \mathrm{p}<0.001)$, whereas $\mathrm{a}_{\bullet}^{\circ}$ significant negative correlation was observed between pyridinoline excretion and grip strength $(\mathrm{p}<0.05)$. No such correlations were noted for deoxypyridinoline.

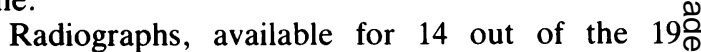
individuals, were assessed using Sharp's method. The highest concentrations of urinary pyridinoline were $\overrightarrow{\overrightarrow{0}}$ found in those assessed as having grade II (mean $\exists$ $150 \cdot 2$; range $47 \cdot 0-224.9 \mathrm{nmol} / \mathrm{mmol}$ creatinine) and grade III (mean $86 \cdot 2$; range $48 \cdot 0-154.0 \mathrm{nmol} / \mathrm{mmol}$ creatinine) and the lowest in those with grade $0 \cong$ (mean 20.6; range 18.0-23.2 nmol/mmol creatinine) $\frac{0}{\sigma}$ radiographic changes.

\section{Discussion}

The results of this study showed significantly higher? concentrations of urinary pyridinoline in patientso with RA, which were strongly associated with the activity of the disease. The concentrations of urinaryo deoxypyridinoline, however, remained normal.n These findings appear, therefore, to be indicative of the joint process known to occur in RA.

A possible source of pyridinoline is the type II and type IX collagens of articular cartilage, but also the type I collagen of bone in which pyridinoline is less abundant; this last tissue is the only contributor of deoxypyridinoline. ${ }^{34}$ It is well recognised that join 6 destruction in RA is associated with two types of bone loss: periarticular bone loss, which is an earlyल radiological finding in RA, associated with the locaP inflammatory process, and generalised bone loss, 

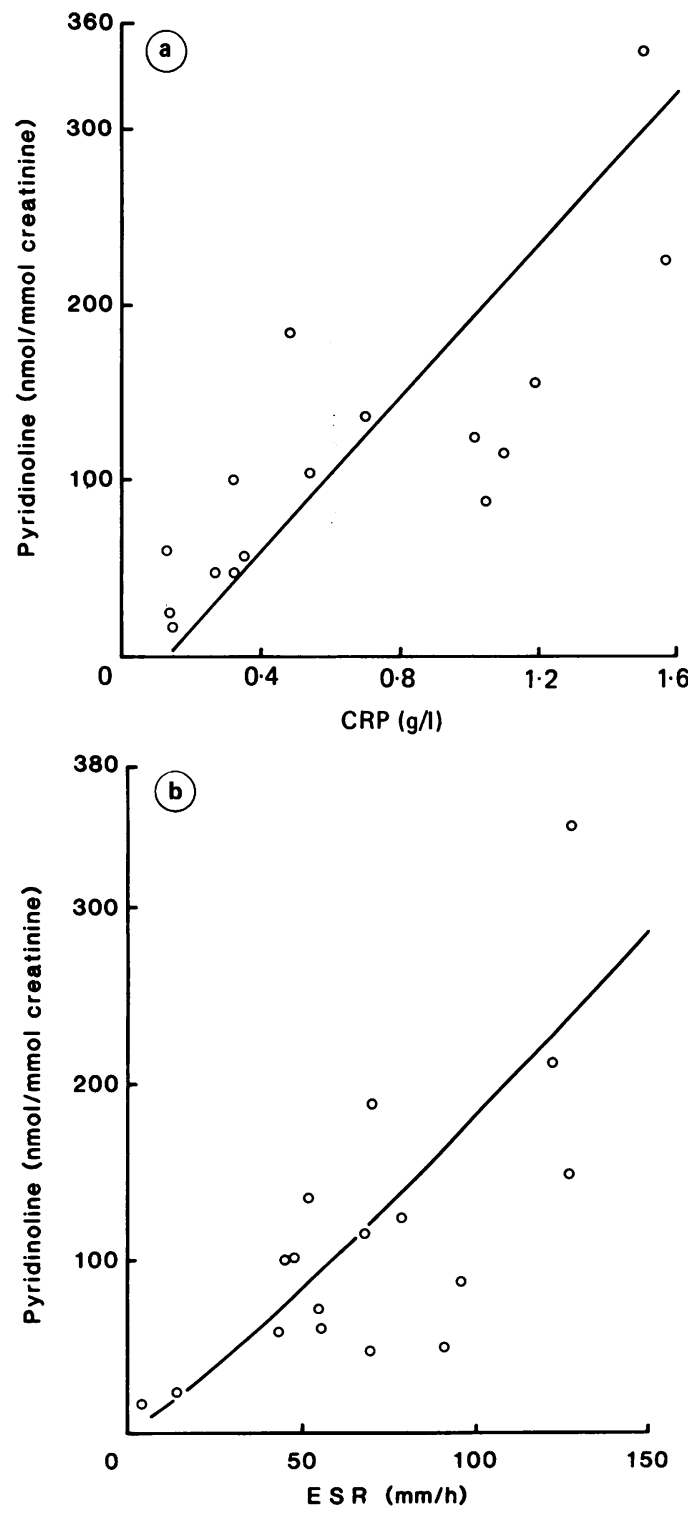

for which the causes and mechanisms are not fully understood. ${ }^{10} 11$ In this study $x$ rays indicated the presence of the former and that this was associated with high concentrations of urinary pyridinoline. No significant increase in urinary deoxypyridinoline was noted, however, despite the fact that significant increases in the concentrations of both pyridinoline and deoxypyridinoline have been observed in conditions where bone turnover and degradation are substantially raised, such as Paget's disease and

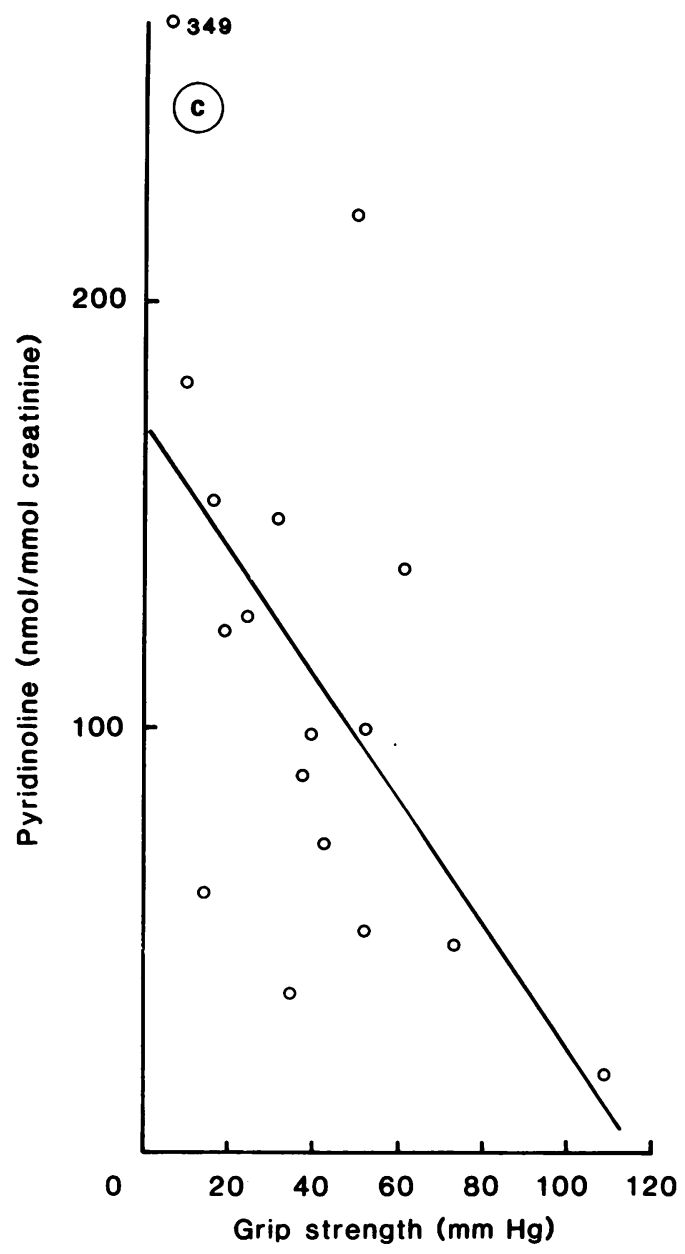

Fig. 1 Correlations of values for urinary pyridinoline measured in patients with rheumatoid arthritis with values for (a) C reactive protein (CRP), (b) erythrocyte sedimentation rate (ESR), and (c) grip strength. The best-fit lines calculated from the data points are shown.

thyrotoxicosis (Black D and Robins $S \mathrm{P}$, unpublished observations). Thus current evidence leads to the conclusion that the increased urinary pyridinoline in patients with RA is due to the loss of collagen matrix primarily from the articular cartilage of the joint and to a lesser extent from the periarticular region of the bone. Preliminary analyses have shown that pyridinoline (but not deoxypyridinoline) is a major cross link in human synovial tissue (Robins S P and Seibel $\mathbf{M} \mathrm{J}$, unpublished observations). The 
possibility should be considered, therefore, that in RA some of the urinary pyridinoline may be derived from the synovium but in the absence of any information on the metabolic turnover of this tissue no estimate of the quantitative contribution can yet be made.

The strong positive correlation between the serum concentrations of CRP and the urinary concentrations of pyridinoline is consistent with the involvement of various cytokines, such as interleukin 1 and interleukin 6 , in the pathogenesis of RA and the association of the destructive process with the acute phase response. It is now well established that interleukin 1 is produced in the synovium of patients with RA, leading to the stimulation of collagenase and other proteinase production by chondrocytes and osteoclasts, which results ultimately in cartilage destruction. ${ }^{12}$ Further, it has been shown that interleukin 1 stimulates release of the hepatocyte stimulating factor interleukin 6 from monocytes, leading to the production of several acute phase proteins, including CRP, by the liver. ${ }^{13} \mathrm{~A}$ recent study has shown the presence of interleukin 6 in the serum and synovium of patients with RA. ${ }^{14}$

The data for pyridinoline and deoxypyridinoline were expressed relative to creatinine in order to incorporate a correction for body mass. Previous studies had shown a lack of correlation between pyridinoline and creatinine excretion, ${ }^{7}$ and, in addition, patients with a history of renal impairment were specifically excluded from this study. The concentrations of pyridinoline for both control and patient samples were about fourfold higher than those obtained in a previous study from this laboratory, ${ }^{7}$ but this discrepancy is likely to be due to underestimation of pyridinoline in the ELISA compared with the fluorimetric detection of HPLC separated cross links used in this study.

In conclusion, the concentrations in urine of the mature collagen cross link pyridinoline are strongly associated with disease activity in patients with RA, reflecting the degradative processes occurring in the rheumatoid joint. Thus measurements of urinary pyridinoline might provide a sensitive, non-invasive biochemical marker for studying the progression and activity of RA. Further studies are necessary, $\overrightarrow{\vec{F}}$ however, to establish the significance of the excretion $\stackrel{\text { ? }}{+}$ of the deoxy analogue in disease states.

We thank the Scottish Home and Health Department for financial support, and Sandy Duncan for skilful technical assistance.

\section{References}

1 Robins S P. An enzyme-linked immunoassay for the collagen. $\vec{\circ}$ crosslink pyridinoline. Biochem J 1982; 207: 617-20.

2 Black D, Duncan A, Robins S P. Quantitative analysis of the $\vec{\omega}$ pyridinium crosslinks of collagen in urine using ion-paired reversed-phase high-performance liquid chromatography. Anal Biochem 1988; 169: 197-203.

3 Eyre D R, Koob J J, Van Ness K P. Quantitation of $\stackrel{\oplus}{\oplus}$ hydroxypyridinium crosslinks by HPLC. Anal Biochem 1984;00 137: $380-8$.

4 Robins S P, Duncan A. Crosslinking of collagen. Biochem $J \stackrel{\nexists}{ \pm}$ 1983; 215: 175-82.

5 Robins S P, Duncan A. Pyridinium crosslinks of bone collagen and their location in peptides isolated from rat femur. Biochim Biophys Acta 1987; 914: 233-9.

6 Reid K B M, Porter R R. Subunit composition and structure of을 subcomponent $\mathrm{Clq}$ of the first component of human comple-es ment. Biochem J 1976; 155: 19-23.

7 Robins S P. Stewart P, Astbury C, Bird H A. Measurement of $\overrightarrow{0}$ the cross linking compound, pyridinoline, in urine as an index of collagen degradation in joint disease. Ann Rheum Dis 1986; 45: 969-73.

8 Sharp J T, Young D Y, Bluhm G B, et al. How many joints in the hands and wrists should be included in a score of radiological abnormalities used to assess rheumatoid arthritis? Arthritis Rheum 1985; 28: 1326-34.

9 Folin A, Wu T. In: Hawk H B, Oser B L, Summerrson W H, eds. Practical physiological chemistry. 12th ed. New York: $\overrightarrow{\bar{A}}$ McGraw-Hill, 1965: 506.

10 Sambrook P N, Reeve J. Bone disease in rheumatoid arthritis Clin Sci 1988; 74: 225-30.

11 Bjilsma J W J. Bone metabolism in patients with rheumatoid arthritis. Clin Rheumatol 1988; 7: 16-23.

12 Murphy G, Reynolds J J. Current views of collagen degradation: progress towards understanding the resorption of connectivetissues. Bioassays 1985; 2: 55-60.

13 Gauldie J, Richards C, Harnish D, Landsdorp P, Baumann $\mathrm{H}_{2}$ Interferon $\beta_{2}$ /BSF- 2 shows identity with monocyte derived hepatocyte stimulating factor (HSF) and regulates the major acute phase protein response in liver cells. Proc Natl Acad Scio USA 1987; 84: 7251-5.

14 Haussiau F A, Devogelaer J-M, Van Damme J, Deuxchaisnes $\mathrm{C} N$, Van Snick J. Interleukin 6 in synovial fluid and serum of patients with rheumatoid arthritis and other inflammatorys arthritides. Arthritis Rheum 1988; 31: 784-8. 